\title{
The flux of interstellar particles detected in the Solar System
}

\author{
Mária Hajduková Jr. \\ Astronomical Institute of the Slovak Academy of Sciences \\ Dúbravská cesta 9, 84505 Bratislava, The Slovak Republic \\ email: astromia@savba.sk
}

\begin{abstract}
The present work shows the proportion of interstellar meteors from different mass ranges (exceeding 20 orders of mass scale) detected by various observational techniques. Having analysed the IAU Meteor Data Center, we find that the mass index of interstellar particles continuously increases towards higher masses, but there is a significant change between $10^{-10}$ $10^{-11} \mathrm{~kg}$. This break is possibly caused by different physical processes leading to different populations of interstellar particles and might be connected with their origin.
\end{abstract}

Keywords. Meteors, meteoroids, interplanetary medium

\section{Observations of interstellar particles by different techniques}

A search for interstellar meteoroids entering the Solar System is currently going on using different techniques, covering a mass scale of more than 20 orders, from faint particles detected by cosmic detectors up to the range of bolides detected by photographic methods. The substantial question of how many interstellar particles there are in comparison with those belonging to our interplanetary cloud has led to many searches. Reports from the Advanced Meteor Orbit Radar (AMOR) in New Zealand and space borne observations, mainly from the Ulysses and Galileo spacecrafts, contradict, in some way, the most precise classical photographic observations as well as the broader photographic data included in the IAU Meteor Data Center (MDC), where only a very few meteors slightly exceeding the hyperbolic velocity limit in the mass range of large particles corresponding to $10^{-4}-10^{1} \mathrm{~kg}$ (Hajduková 1994, 2008).

The orbital and geophysical data of the latest version of MDC, containing 4,581 photographic and 62,906 radar meteors (Lindblad et al. 2005), has been analysed. As detailed analysis showed, the vast majority of the hyperbolic orbits are a consequence of measurement errors in the determination of meteor velocity. We obtained values for interstellar meteor fluxes of $6 \times 10^{-14} \mathrm{~m}^{-2} \mathrm{~s}^{-1}$ for radar data $\left(m>7 \times 10^{-8} \mathrm{~kg}\right)$ and $7 \times 10^{-19} \mathrm{~m}^{-2} \mathrm{~s}^{-1}$ for photographic meteors $m>10^{-3} \mathrm{~kg}$.

Hawkes \& Woodworth (1997a) obtained a contribution of hyperbolic meteors at the level of $1-2 \%$ of the mass range between $10^{-4}-10^{-9} \mathrm{~kg}$ using a video detector technique. Mathews et al. (1999), using an ultra high frequency (UHF) radar technique, detected a small level of hyperbolic particles in the mass range of $10^{-9}-10^{-12} \mathrm{~kg}$, reaching the upper mass limit of space-born particles. Weryk \& Brown (2005) using the Canadian Meteor Orbit Radar (CMOR) orbital data obtained the flux of interstellar meteoroids arriving at the Earth of $6 \times 10^{-6}$ meteoroids $\mathrm{km}^{-2} \mathrm{~h}^{-1}$ for the mass of $1 \times 10^{-8} \mathrm{~kg}$.

The question arising from the above results is whether it is possible to bring all the above mentioned data to a common view of the real contribution of interstellar particles across the broad scale of mass, exceeding 20 orders of magnitude, or whether we should search for errors in the results or methods leading to them? 


\section{The flux and mass distribution of interstellar particles}
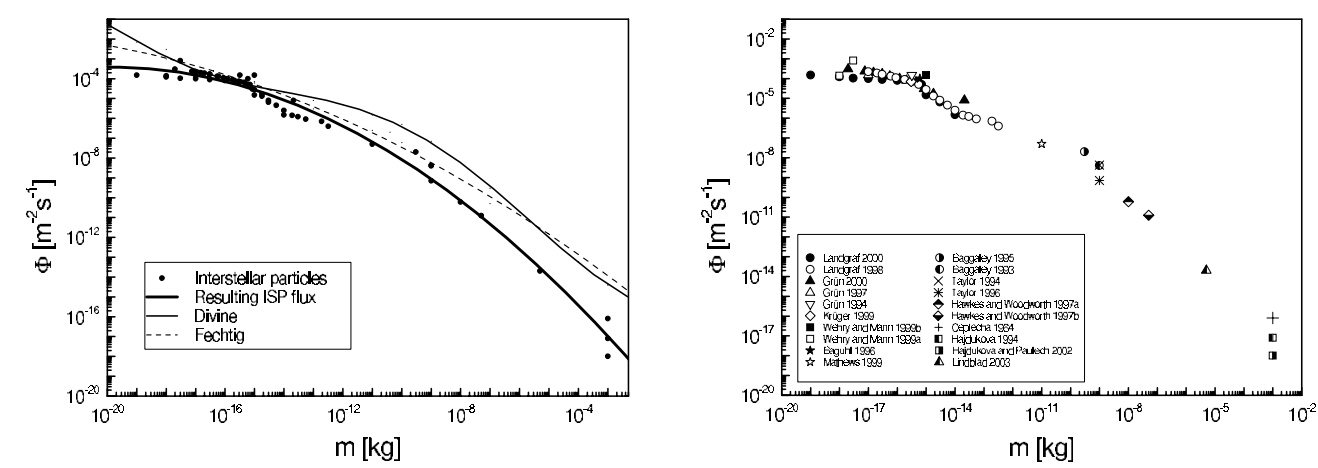

Figure 1. Flux of interstellar particles along the mass range (right panel), from observations by different techniques, in comparison with the flux of interplanetary particles according to Divine (solid line) and Fechtig (dashed line) (left panel).

Figure 1 is constructed from the results of different authors, as well as from our obtained values of interstellar meteor flux from the MDC. The sources of the data are given in the right side of the figure. The heavy line in Figure 1 (left) represents a second order polynomial of interstellar fluxes, over the whole mass scale from all given data. The fainter lines represent the second order polynomial for the interplanetary flux data given by Divine et al. (1993) (solid line) and Fechtig (1976) (dashed line). As is seen in Figure 1, the flux of interstellar particles is more than 2 orders of magnitude lower than the flux of interplanetary ones in the mass range of large particles, but it increases towards the smaller particles. Their mass distribution is steeper, however the critical value of the mass index $s_{i s}=2$ corresponds to almost the same mass interval, between $10^{-10}-10^{-11} \mathrm{~kg}$. The mass index changes continuously, increasing towards higher masses. The data implies 3 distinct mass intervals, however a substantial change of $s$ values occurs between $10^{-11}$ $10^{-10} \mathrm{~kg}$ as a break in the interstellar flux distribution. This break is possible caused by different physical processes leading to population of interstellar particles and might be connected with their origin (Hajduková \& Hajduk 2006).

\section{Acknowledgements}

This work was supported by the Slovak Scientific Grant Agency VEGA, grant No 3067.

\section{References}

Divine, N., Grün, E., \& Staubach, P. 1993, in: W. Flury, Darmstadt (eds.), Proceeding of the First European Conference on Space Debris, ESA SD - 01, p. 245

Fechtig, H. 1976, in: C. L. Hemenvay, P. M. Millman and A. F. Cook (eds.), Evolutionary and Physical Properties of Meteoroids, NASA SP, 319, p. 218

Hajduková, M. Jr. 1994, A\&̊A, 288, 330

Hajduková, M. Jr. 2008, Earth, Moon, Planets, 102, 67

Hajduková, M. Jr. \& Hajduk, A. 2006, Contri. Astron. Obs. Skalnaté Pleso, 36, 15

Hawkes, R. L. \& Woodworth, S. C. 1997a, J. R. Astron. Soc. Can., 91, 68

Hawkes, R. L. \& Woodworth, S. C. 1997b, J. R. Astron. Soc. Can., 91, 218

Lindblad, B. A., Neslušan, L., Porubčan, V., \& Svoreň, J. 2005, Earth, Moon, Planets, 93, 249

Mathews, J. D. et al. 1999, in: W. J. Baggaley and V. Porubčan (eds.), Meteoroids 1998, (Slovak Academy of Sciences, Bratislava), p. 79

Weryk, R. J. \& Brown, P. 2005, Earth, Moon, Planets 95, 221 